\title{
Selective Reward Deficit in Mice Lacking $\beta$-Endorphin and Enkephalin
}

\author{
Michael D. Hayward, ${ }^{1}$ John E. Pintar, ${ }^{3}$ and Malcolm J. Low ${ }^{1,2}$ \\ 1 The Vollum Institute and 2Department of Behavioral Neuroscience, Oregon Health and Science University, Portland, \\ Oregon 97201, and ${ }^{3}$ Robert Wood Johnson Medical School, Department of Neuroscience and Cell Biology, Piscataway, \\ New Jersey 08854
}

It has been impossible to unequivocally identify which endogenous opioids modulate the incentive value of rewarding stimuli because these peptides are not highly selective for any single opioid receptor subtype. Here, we present evidence based on the measurement of instrumental behavior of $\beta$-endorphin and enkephalin knock-out mice that both opioid peptides play a positive role. A progressive ratio schedule was used to measure how hard an animal would work for food reinforcers. The loss of either opioid reduced responding under this schedule, regardless of the palatability of the three different formulas of reinforcers used. The phenotype of mice lacking both endogenous opioids was nearly identical to the phenotype of mice mutant for either individual opioid. Responses were tested in nondeprived and deprived feeding states but were reduced in $\beta$-endorphin- and enkephalin-deficient mice only when they were maintained under nondeprived conditions. Other operant manipulations ruled out variables that might contribute nonspecifically to this result such as differences in acquisition, early satiation, motor performance deficit, and reduced resistance to extinction. In contrast to the effects on instrumental performance, the loss of either or both endogenous opioids did not influence preference for water flavored with sucrose or saccharin in a two-bottle free-choice drinking paradigm. We conclude that both $\beta$-endorphin and enkephalin positively contribute to the incentive-motivation to acquire food reinforcers. Because the attenuation of operant responding was observed only during a nondeprived motivational state, the hedonics of feeding are likely altered rather than energy homeostasis.

Key words: endogenous opioids; reinforcement; operant conditioning; mouse; feeding; motivation
The endogenous opioid system influences incentive-motivation in a number of different tests. Naloxone, a non-subtype-selective opiate receptor antagonist, reduces consumption of a variety of palatable foods and decreases operant responding for food reinforcers (for review, see Morley, 1987; Glass et al., 1999a). The endogenous opioid system also modulates self-administration of alcohol, benzodiazepines, psychostimulants, narcotics (for review, see Van Ree et al., 1999), and intracranial electrical selfstimulation (Trujillo et al., 1989). In fact, a role for enkephalin in reward behavior was suggested soon after the first identification of an endogenous opioid (Beluzzi and Stein, 1977). Thus, a general role for the endogenous opioid system may be to enhance the incentive value of rewarding stimuli.

Many previous experiments have studied the role of the endogenous opioid system in reward-related behaviors by using subtype-selective opioid receptor antagonists. However, the endogenous opioid peptides interact relatively nonspecifically with the different opioid receptors (Reisine and Pasternak, 1996), making it difficult to draw conclusions regarding which endogenous opioids are involved in behaviors such as positive reinforcement. $\beta$-Endorphin has nearly equal affinity for the $\mu$ and $\delta$ opioid receptor and enkephalin preferentially binds to the $\delta$ receptor,

\footnotetext{
Received April 1, 2002; revised June 10, 2002; accepted June 14, 2002.

This work was funded by National Institutes of Health Grants DA05841 (M.D.H.), DA09040 (J.E.P.), DA14203, and DK55819 (M.J.L.). We thank Drs. Chris Cunningham and Fred Risinger for useful discussions concerning operant behavior and conditioning and Drs. Shane Hentges, Greg Mark, and Suzanne Mitchell for helpful comments on this manuscript.

Correspondence should be addressed to Dr. Malcolm J. Low, Vollum Institute L-474, Oregon Health and Science University, 3181 Southwest Sam Jackson Park Road, Portland, OR 97201. E-mail: low@ohsu.edu.

Copyright (C) 2002 Society for Neuroscience $0270-6474 / 02 / 228251-08 \$ 15.00 / 0$
}

although it also has physiologically relevant affinity for the $\mu$ receptor (Reisine and Pasternak, 1996). Agonists for all three opioid receptors can stimulate feeding to varying degrees, but agonists for the $\mu$ and $\delta$ receptor are thought to be intrinsically rewarding, whereas agonists for the $\kappa$ receptor have been shown to actually be aversive (Mucha and Herz, 1985; Bals-Kubik et al., 1989; Székely, 1994). Thus, $\beta$-endorphin and enkephalin are the most likely opioid peptides involved in positively reinforced operant behavior. To test the function of these specific endogenous opioid peptides in mice, we mutated the pro-opiomelanocortin (POMC) gene so that it does not express $\beta$-endorphin (Rubinstein et al., 1996) and the preproenkephalin gene so that none of the enkephalin peptides are made (Ragnauth et al., 2001) (J. N. Nitsche, A. G. P. Schuller, M. A. King, M. Zheng, G. W. Pasternak, and J. E. Pintar, unpublished observations).

We tested for changes in the incentive value of rewarding stimuli by quantifying the reinforcing efficacy of food pellets using operant responding under a progressive ratio (PR) schedule, which requires additional bar presses of a defined number for each subsequent reinforcer (Hodos, 1961). PR schedules have been widely used to quantify the value an animal places on a commodity by measuring the effort they will expend to receive that reinforcer. In fact, this procedure has been successfully used to measure naloxone effects on motivation to obtain food reinforcers in rats and mice (Rudski et al., 1994; Cleary et al., 1996; Glass et al., 1999b; Hayward and Low, 2001). The incentive value of food varies with motivational states such that the neurobiological substrate underlying the instrumental behavior may be different in freely fed states from food-deprived states (Rudski et al., 1994; Nader et al., 1997). For example, the hedonic value of food may be the primary motivator in food-reinforced operant behav- 
ior under free-feeding conditions. Caloric imbalance would be the significant contributor to the incentive value of food reinforcers under food-deprived conditions. Here, we present the first evidence that loss of the $\beta$-endorphin or enkephalin peptides results in reduced motivation to acquire food reinforcers selectively in a nondeprived state, one in which the hedonic value of feeding likely predominates over energy state.

\section{MATERIALS AND METHODS}

\section{Subjects}

The $\beta$-endorphin-deficient mice were previously described, and complete loss of $\beta$-endorphin expression was demonstrated (Rubinstein et al., 1996). Likewise, the enkephalin-deficient mice were previously described (Ragnauth et al., 2001), and complete loss of Met-enkephalin and Leuenkephalin peptides was demonstrated (Nitsche, Schuller, King, Zheng, Pasternak, and Pintar, unpublished observation). The two mutant gene alleles on chromosomes 13 and 4, respectively, were backcrossed simultaneously onto the $\mathrm{C} 57 \mathrm{BL} / 6 \mathrm{~J}$ genetic background for five generations $\left(\mathrm{N}_{5}\right)$ using double heterozygous mice and alternating genders for each generation. The mice used for all behavioral experiments were sibling offspring of double heterozygous $\mathrm{N}_{5}$ intercrosses and were individually genotyped by PCR using $100 \mathrm{ng}$ of genomic DNA and 1-2 $\mu \mathrm{M}$ deoxyoligonucleotide primer trios in a total volume of $30 \mu \mathrm{l}$. The primer sequences for the POMC alleles were: POMC exon 3 sense, 5'-GAA GTA CGT CAT GGG TCA CT-3'; POMC 3' flanking antisense, 5'-GCT GGG GCA AGG AGG TTG AGA-3'; and PGKNeo sense, 5'-GAG GAT TGG GAA GAC GAC AAT AGC A-3'. The primer sequences for the proenkephalin alleles were: proenkephalin exon 3 sense, $5^{\prime}$-AAT GAC GAA GAC GAA GAC ATG AGC AAG A-3'; proenkephalin exon 3 antisense, 5'-CAT CCA GGA GAG ATG AGG TAA C-3'; and PMCNeo antisense, 5'-CTC GAC ATT GGG TGG AAA CAT TCC-3'. The touchdown reaction conditions for each trio of primers included denaturation at $94^{\circ} \times 1 \mathrm{~min}$, then 15 cycles of annealing at $T_{\mathrm{m}} 67^{\circ} \times 1$ min with a decrease by $1 \%$ cycle and extension at $72^{\circ} \times 1 \mathrm{~min}$. The final 15 cycles all used an annealing temperature of $52^{\circ}$ and extension at $72^{\circ} \times$ $1 \mathrm{~min} /$ cycle. The amplification products were $\sim 700 \mathrm{bp}$ for the End ${ }^{+}$ allele, $\sim 100 \mathrm{bp}$ for the End ${ }^{-}$allele, $\sim 500 \mathrm{bp}$ for the Enk ${ }^{+}$allele, and $\sim 300$ bp for the Enk ${ }^{-}$allele.

The same groups of male wild-type $\left(\mathrm{Enk}^{+/+}, \mathrm{End}^{+/+} ; n=9\right)$, $\beta$-endorphin-deficient $\left(\right.$ Enk $^{+/+}$, End $\left.^{-/-} ; n=10\right)$, enkephalin-deficient $\left(\right.$ Enk $^{-/-}$, End $\left.^{+/+} ; n=10\right)$, and double knock-out (Enk ${ }^{-1-}$, End $^{-1-} ; n=$ 6) mice were used for all of the experiments described. The subjects ranged in ages from 3 to 4 months at the outset of the study and were $12-18$ months of age at the termination of the study. One subject was found dead in his home cage near the end of the extinction experiment, so data from that animal were not included in the extinction results. Foodrestricted conditions were monitored by daily weighing of mice, and the amount of food was adjusted to maintain body weights during training at $75-85 \%$ of initial weight, and during deprivation testing at $75-85 \%$ of weight while previously feeding ad libitum. Water was always available ad libitum, and subjects were housed in a 14:10 light/dark cycle with lights on at 5:00 A.M. All procedures were approved by the Institutional Animal Care and Use Committee and followed the Public Health Service guidelines for the humane care and use of experimental animals.

\section{Apparatus}

Four mouse operant conditioning chambers (MedAssociates, St. Albans, VT) were used. Each chamber consisted of a $16.5 \times 14 \mathrm{~cm}$ metal grid floor with Plexiglas walls and ceiling contained in a sound attenuated and ventilated cabinet. During testing the subjects were presented with two retractable ultrasensitive levers, one on either side of a tray into which pellets were dispensed from an elevated hopper. Completion of the instrumental contingency on the left lever (i.e., active lever) resulted in the presentation of one pellet in the food hopper. Above the inactive lever was a yellow light-emitting diode (LED), the only light source in the chamber. When sessions began the levers were extended, and the LED was illuminated, and when sessions ended the LED was turned off and both levers were retracted. The three formulas used for the $20 \mathrm{mg}$ reinforcement pellets were a "normal chow" (Formula A/I; P.J. Noyes Co., Lancaster, $\mathrm{NH}$ ), which is similar to the animal's normal maintenance chow (5\% fat, $19 \%$ protein, and $5 \%$ fiber; $3.4 \mathrm{kcal} / \mathrm{gm}$ ), a "sweet chow" composed of sucrose with binder (Formula F; P.J. Noyes Co.), and a "fat chow" (pellets were custom-made by P.J. Noyes Co. from PicoLab
Mouse Diet 20; PMI Feeds Inc., St. Louis, MO), which is isocaloric to the "normal chow" but contains twice the amount of fat.

\section{Behavioral assays}

Operant behavior. The shaping protocol was as follows: (1) All subjects experienced $5 \mathrm{~d}$ of restricted food access to maintain each animal between $75-85 \%$ of their original body weight. To avoid food neophobia, $30 \%$ of the daily food access included an equal part mixture of all three formulas of reinforcers during the food-restricted portion of shaping. This restricted daily access to food was enforced during the following steps $2-4$.

(2) Two to four sessions under a fixed ratio (FR) 1 schedule with only the active lever and priming with a single food reinforcer before each 30 min session. Mice that pressed the active lever $>10$ times were advanced to the next stage, and those that did not press the active lever more than ten times during the fourth session were excluded [only one subject with genotype of (Enk ${ }^{-/-}$, End ${ }^{-/-}$) was excluded].

(3) Two sessions under an FR1 schedule with the inactive lever introduced during the 30 min sessions

(4) Two to four sessions under an FR5 (30 min sessions with active and inactive lever).

(5) Food was returned to the home cage, and ad libitum feeding was reestablished for $4 \mathrm{~d}$ with no operant sessions.

(6) Two to four sessions under an FR5 while feeding ad libitum (30 min sessions)

(7) Six sessions under a PR3 while feeding ad libitum to stabilize behavior.

Data gathering from the PR3 sessions then began. The number of shaping FR sessions varied slightly during steps 4 and 6 so that al subjects in a testing cohort began the PR3 sessions on the same day. The PR3 schedule increased the number of lever presses by three for each subsequent reinforcer, i.e., the first reinforcer required 3 lever presses, the second reinforcer required 6 , the third reinforcer required 9 , etc. $(3 n+3$ where $n=$ number of reinforcers received). The breakpoint was defined as the last ratio level completed before 15 min elapsed without the mouse receiving a reinforcer; i.e., subjects had $15 \mathrm{~min}$ to complete each ratio level. Experimental sessions were conducted once each day for each animal and took place between 10:00 A.M. and 6:00 P.M. The start-time was randomized so that no subject was tested at the same time each day. All mice were tested under all treatment conditions in a within-subjects design, alternating chow formula (normal, sweet, and fat) daily and balancing for genotype. Each condition was measured five times for each animal as a repeated measure. This $3 \mathrm{~d}$ cycle was first completed under ad libitum feeding conditions (a total of 15 sessions for each subject) as a single block and then repeated in the food-restricted state (total of 15 sessions for each subject) as a single block. During the food-restricted portions of the study, mice were fed at the end of the day after all operant sessions had ended ( 6:00 P.M.). A $10 \mathrm{~d}$ interval elapsed between the two testing blocks to allow for a stable reduction of weight under food restriction. After all of the PR3 tests, mice were allowed to return to ad libitum feeding conditions for at least $10 \mathrm{~d}$ before they were retested under an FR5 schedule. Three initial $1 \mathrm{hr}$ sessions were conducted, once for each chow formula, for a period of equilibration. FR5 sessions were then conducted using three sessions for each chow formula, alternating chow formula daily and balancing for genotype. Extinction sessions were conducted using the PR3 schedule in the same chambers, but the pellet dispensers were inactivated, and there were no food reinforcers delivered. A total of eight extinction sessions were conducted, and no subject was tested more than once each day. Subjects were always tested in the same chamber for all operant studies.

Two bottle free choice. The individually housed subjects were presented with two graduated cylinders equipped with lixit tubes in their home cages. Tap water was first presented in both bottles for $4 \mathrm{~d}$ to acclimate subjects to the apparatus. The two-bottle free choice paradigm consisted of $4 \mathrm{~d}$ cycles with mice successively having access to tap water, sucrose (2, 4,8 , and $16 \%$ ) and sodium saccharin $(0.01,0.1,1$, and $10 \%)$, always versus tap water in the second bottle. Twenty-five milliliter graduated cylinder drinking tube positions were reversed every $2 \mathrm{~d}$ to control for side preferences, and the second day's reading on each side was used. Two measurements of fluid consumption were taken for each tastant concentration. Sucrose consumption was measured over $6 \mathrm{hr}$ beginning 2 hr after light onset because all subjects in overnight experiments consumed all $25 \mathrm{ml}$ of solution. Saccharin consumption was measured over $24 \mathrm{hr}$. Consumption of tap water controls for both tastants was measured over $24 \mathrm{hr}$. Leakage was adjusted using an empty control cage that was 


\begin{tabular}{|c|c|c|c|c|c|c|c|}
\hline $\begin{array}{l}\text { Test } \\
\text { genotype }\end{array}$ & Shaping & $\begin{array}{l}\text { PR3 } \\
\text { ad libitum }\end{array}$ & $\begin{array}{l}\text { PR3 food- } \\
\text { restricted }\end{array}$ & $\begin{array}{l}\text { Sucrose- } \\
\text { choice }\end{array}$ & $\begin{array}{l}\text { Saccharin- } \\
\text { choice }\end{array}$ & $\begin{array}{l}\text { FR5 } \\
\text { ad libitum }\end{array}$ & Extinction \\
\hline $\mathrm{Enk}^{+/+}, \mathrm{End}^{+/+}$ & $3-8$ & $4-9$ & $5-11$ & $9-15$ & $10-16$ & $10-17$ & $12-18$ \\
\hline $\mathrm{Enk}^{+/+}, \mathrm{End}^{-/-}$ & $3-6$ & $4-7$ & $5-9$ & $9-12$ & $10-14$ & $11-16$ & $12-18$ \\
\hline $\mathrm{Enk}^{-/-}, \mathrm{End}^{+/+}$ & $3-9$ & $4-10$ & $5-12$ & $7-14$ & $8-15$ & $9-16$ & $11-18$ \\
\hline $\mathrm{Enk}^{-/-}, \mathrm{End}^{-/-}$ & $3-5$ & $4-6$ & $5-8$ & $9-12$ & $10-13$ & $10-15$ & $12-17$ \\
\hline
\end{tabular}

handled at the same time. Food consumption was also measured daily by weighing the amount of food remaining in each cage and subtracting from the food weight of the previous day.

\section{Experimental design and statistical analysis}

The studies were conducted sequentially in the order depicted in Table 1 for all subjects and used a within-subjects, random block design with repeated measures. The dependent variables for the operant paradigms were breakpoint (last ratio completed for a reinforcer), number of reinforcers earned, and number of reinforcers eaten. We counted all of the pellets remaining in the chamber after a session, and a pellet was considered eaten if over half of it was consumed (Hayward and Low, 2001). The ad libitum and food-restricted conditions were analyzed using separate repeated measures ANOVA (RMANOVA). Breakpoints could be analyzed by RMANOVA without violating the assumption of equal variance because the PR3 used was a linear function $(3 n+3)$, and so variance was equal at all points of the scale. All significant differences identified using breakpoint as the dependent variable were recapitulated by RMANOVA using pellets received as the dependent variable (analyses not shown). The sucrose and saccharin drinking data were reported as preference ratios, calculated as the amount of tastant consumed divided by the total fluid intake, and analyzed by RMANOVA. Post hoc simple main effect analyses and pairwise comparisons were performed with Fisher's PLSD when the initial $P$ value was significant. All data were analyzed with StatView 5 for Macintosh (SAS Institute Inc., Cary, NC). Significance was set at $p<0.05$.

\section{RESULTS}

Mice with the four compound genotypes $\left(\mathrm{Enk}^{+/+}, \mathrm{End}^{+/+}\right.$; $\mathrm{Enk}^{+/+}, \mathrm{End}^{-/-} ; \mathrm{Enk}^{-/-}, \mathrm{End}^{+/+} ; \mathrm{Enk}^{-/-}, \mathrm{End}^{-/-}$) were trained to lever press for food reinforcers in an operant conditioning chamber, initially under restricted feeding conditions. This training period consisted of a number of FR sessions first under an FR1, then under an FR5 before introducing the PR schedule (see Materials and Methods for detailed description). During the FR portion of the training period under restricted feeding conditions (steps 2 through 4 given in Materials and Methods), the total number of reinforcers earned did not differ significantly among genotypes (Fig. 1a). No main effect of genotype was found by one-factor ANOVA $\left(F_{(3,31)}=0.9\right.$, NS). Similarly, response rates on both active and inactive levers during the final FR5 session under ad libitum feeding conditions (step 6 given in Materials and Methods) did not vary among genotypes (Fig. 1b). No main effect of genotype was detected by one-factor ANOVA on the active lever $\left(F_{(3,31)}=0.3, \mathrm{NS}\right)$ or inactive lever $\left(F_{(3,31)}=0.9, \mathrm{NS}\right)$. Thus, all of the subjects performed at the same level before being introduced to the PR3 schedule.

We compared the reinforcement efficacy among the four genotypes by conducting PR3 sessions on ad libitum feeding subjects and measured breakpoints for each of three formulas of reinforcers: normal, sweet, and fat chow (composition is given in Materials and Methods). A main effect of genotype was detected by RMANOVA $\left(F_{(3,31)}=3.7, p=0.02\right)$. Breakpoints were significantly higher for wild-type mice responding for all three formulas of reinforcers compared with each of the three mutant genotypes (Fig. 2a). Breakpoints of the mutant genotypes for the

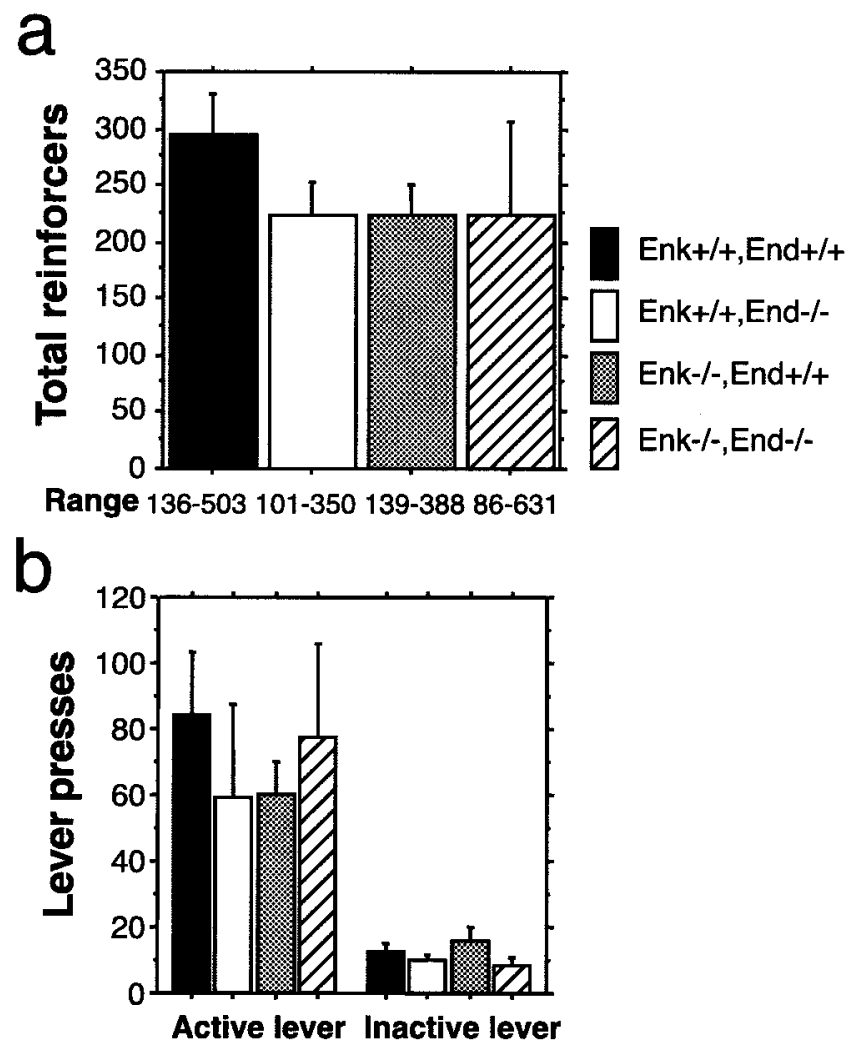

Figure 1. Shaping of operant behavior. $a$, Total number of reinforcers earned $( \pm$ SEM) under the restricted feeding FR portion of the training period (both FR1 and FR5). The range of total reinforcers earned for each genotype is given below the bars. $b$, Number of active and inactive lever presses ( \pm SEM) during the final FR5 session of the training period while feeding ad libitum.

normal and fat chow reinforcers were only approximately half that of the wild-type mice. A main effect of chow formula was detected by RMANOVA $\left(F_{(2,31)}=40.8, p<0.001\right)$, and a chow formula by genotype interaction was also detected $\left(F_{(6,31)}=3.2\right.$, $p=0.008)$. However, separate RMANOVAs conducted on individual genotypes detected main effects by chow formula for three of the genotypes and a strong trend for the double null mutant mice (RMANOVA Enk ${ }^{+/+}$, End $^{+/+}: F_{(2,8)}=27.5, p<0.0001$; $\mathrm{Enk}^{+/+}, \mathrm{End}^{-/-}: F_{(2,9)}=6.7, p=0.007 ; \mathrm{Enk}^{-/-}, \mathrm{End}^{+/+}$: $F_{(2,9)}=10.8, p=0.0008 ; \mathrm{Enk}^{-/-}, \mathrm{End}^{-/-}: F_{(2,5)}=4.0, p=$ $0.053)$. Thus, the rank order of preference was the same in all genotypes (fat chow $>$ normal chow $>$ sweet chow), but the level of behavior supported by these reinforcers differed among genotypes.

For mice in a nondeprived state, the endogenous opioids clearly modulated the efficacy of food reinforcers. However, in a food-deprived state induced by restricted access, the relative reinforcer efficacy was indistinguishable among the four geno- 

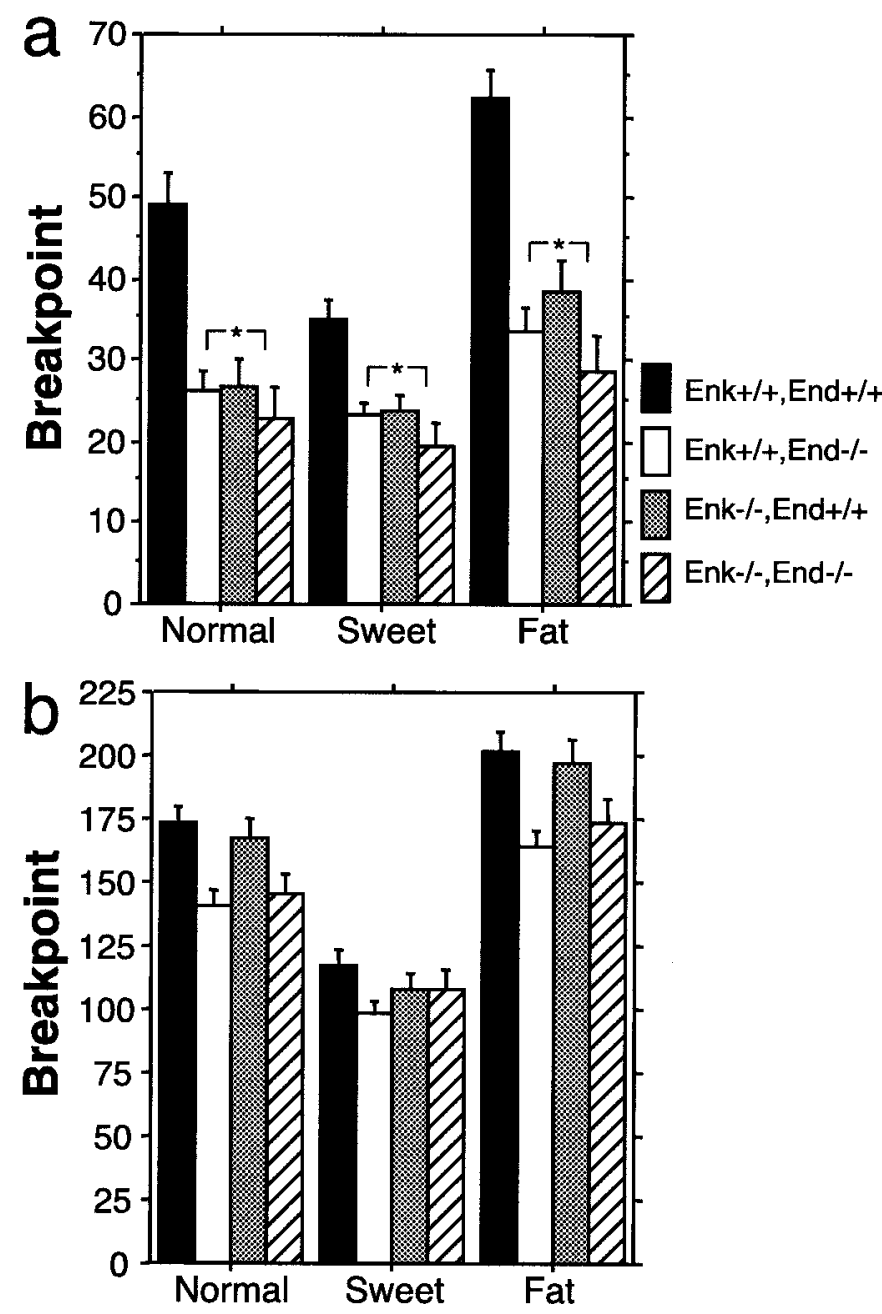

Figure 2. Breakpoints under a PR3 schedule. $a$, Breakpoints $( \pm$ SEM $)$ are given for mice under ad libitum feeding conditions for each of the three formulas of reinforcers. Note that the wild-type mice had significantly higher breakpoints than any of the three mutant opioid genotypes $\left({ }^{*} p<\right.$ 0.0001 by Fisher's PLSD post hoc). $b$, Breakpoints ( \pm SEM) are given for mice under food-restricted conditions for each of the three formulas of reinforcers. Note that there is no difference among the four genotypes.

types (Fig. 2b). Breakpoints did not differ significantly and no main effect of genotype was detected under a PR3 schedule when the mice were given restricted access to food (RMANOVA, $F_{(3,31)}=1.7$, NS). However, a main effect of chow formula was still detected (RMANOVA, $\left.F_{(2,31)}=159.0, p<0.0001\right)$. When separate RMANOVAs were conducted on individual chow formulas there was still no main effect of genotype (RMANOVA, normal chow: $F_{(3,31)}=2.9$, NS; sweet chow: $F_{(3,31)}=0.7$, NS; fat chow: $\left.F_{(3,31)}=1.6, \mathrm{NS}\right)$. All four genotypes responded more for the fat chow reinforcer when access to food was restricted, and this change appeared to be similar for all four genotypes because no genotype by chow formula interaction was detected $\left(\right.$ RMANOVA, $\left.F_{(6,31)}=1.2, \mathrm{NS}\right)$. The rank order of preference was the same as in the ad libitum fed condition, but breakpoints were substantially higher under the food-restricted condition, and there was no significant difference among the four genotypes. This finding supports our argument that the difference in Figure $2 a$ was not caused by a motor deficit and suggests a statedependent difference in motivation.

Under the free-feeding condition, a difference in satiation among the genotypes could confound our interpretation of the PR3 data; so we examined operant behavior under a FR5, a relatively unchallenging and consistent schedule. The average duration of a PR3 session under ad libitum feeding conditions was $\sim 1 \mathrm{hr}$ (data not shown), so we used an FR5 schedule for $1 \mathrm{hr}$ to compare to the data gathered under a PR3 during ad libitum feeding conditions. No main effect of genotype was detected for the number of pellets received under an FR5 schedule when mice were fed ad libitum (RMANOVA, $F_{(3,31)}=1.3$, NS), nor was there a chow formula by genotype interaction (RMANOVA, $F_{(6,31)}=1.3$, NS) (Fig. 3a). However, a main effect of chow formula was detected $\left(F_{(2,32)}=19.6, p<0.0001\right)$ with rank order
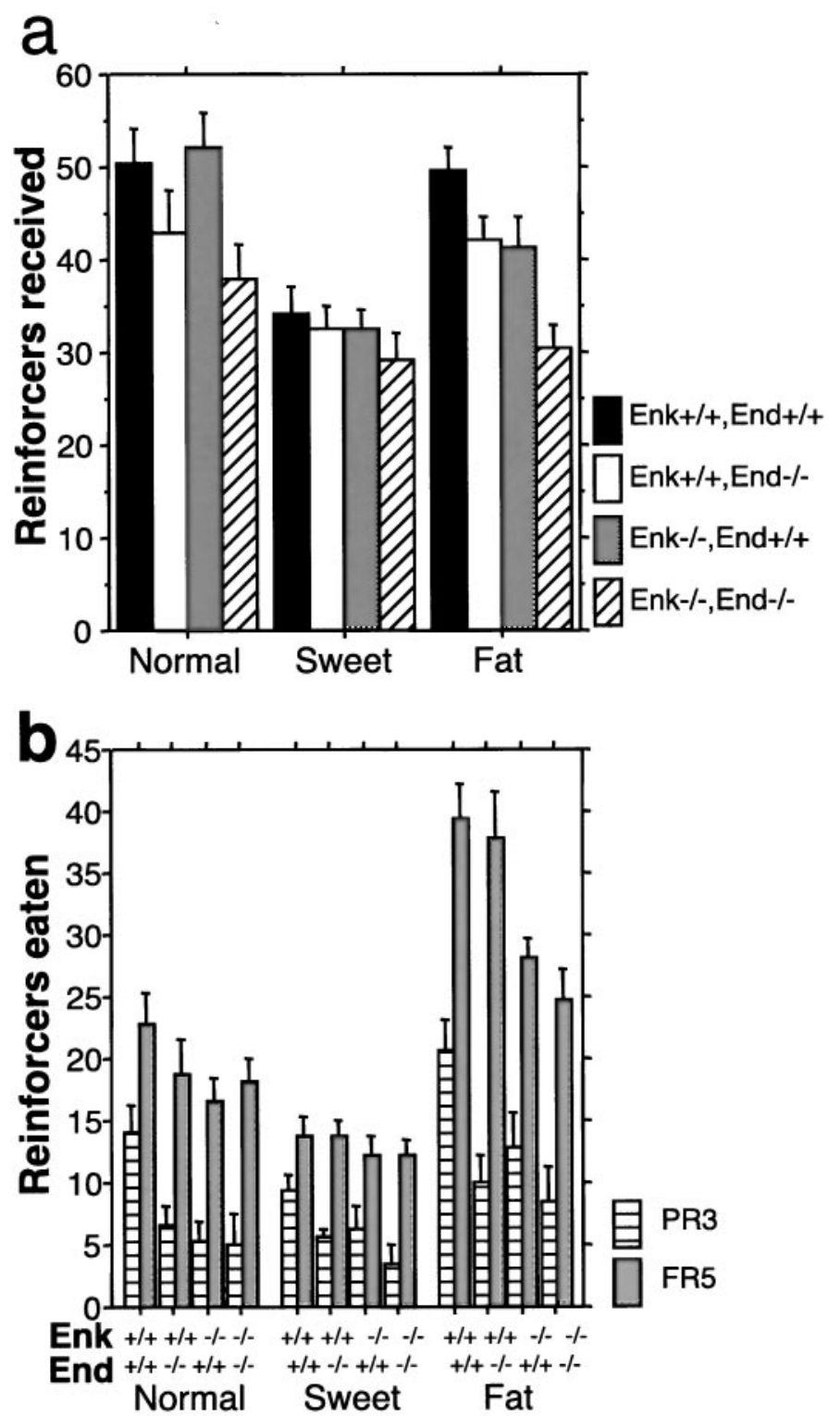

Figure 3. Response rates and feeding under an FR5 schedule. $a$, The number of reinforcers received $( \pm$ SEM) during 1 hr FR5 sessions is given for mice under ad libitum feeding conditions for each of the three formulas of reinforcers. No main effect of genotype was detected. $b$, The number of reinforcers eaten $( \pm \mathrm{SEM})$ during $1 \mathrm{hr}$ FR5 sessions is compared with the number of reinforcers eaten during PR3 sessions while feeding ad libitum. The data compare the two schedules side by side for each genotype and for each formula of reinforcer. Note that the number of reinforcers eaten during FR5 sessions is much greater than during PR3 sessions. 
of preference for the different reinforcers the same as that found in the PR3 sessions. In addition to the number of pellets received, we also measured the number of those earned pellets eaten during the $1 \mathrm{hr}$ FR5 sessions and compared these data to the PR3 sessions performed under ad libitum feeding conditions. During a FR5 schedule the mice from all four genotypes ate significantly more reinforcers of all three formulas than during their PR3 testing (Fig. 3b). A main effect of schedule was detected by RMANOVA conducted on these data $\left(F_{(1,31)}=150.6, p<\right.$ $0.0001)$. This is unlikely to be an artifact of increased workload under the PR3 schedule because the average number of lever presses under the PR3 was only approximately twice that under the FR5 (e.g., active lever presses by Enk ${ }^{+/+}$, $\mathrm{End}^{+/+}$for normal chow: PR3 $=497.6 \pm 34.5$ vs FR5 $=253.6 \pm 8.1)$. These data demonstrated that mice of all four genotypes could eat significantly more pellets in $1 \mathrm{hr}$ than they ate during the PR3 sessions under ad libitum feeding conditions, suggesting that the mutant opioid genotypes did not satiate earlier than wild-type mice.

Because a PR uses an extinction criterion as an endpoint, we also examined the resistance to extinction by the four genotypes. Extinction sessions were conducted when the mice were feeding ad libitum using the PR3 schedule and a 15 min limit to reach the next ratio, but with no reinforcers (PR3-EXT). The endpoint for extinction trials was determined by a post hoc analysis and a criterion of 3 consecutive days that were not significantly different from each other (days 6-8, data not shown). Extinction curves were generated using the data from days 1 through 6 (Fig. 4a). Main effects were detected for the breakpoints among genotypes (RMANOVA, $F_{(3,30)}=3.9, p=0.02$ ) and extinction day (RMANOVA, $\left.F_{(5,30)}=78.1, p<0.0001\right)$. Importantly, there was no day by genotype interaction $\left(F_{(15,30)}=1.2, \mathrm{NS}\right)$, so it appeared that all genotypes extinguished at the same rate. Simple main effects and post hoc analyses conducted on individual days did not detect any consistent differences among any of the mutant opioid genotypes and wild-type mice (day one, $F_{(3,30)}=2.2$, NS; day two, $F_{(3,30)}=3.2, p=0.04$, Fisher's PLSD post hoc significant difference between Enk ${ }^{+/+}$, End $^{-/-}$and Enk ${ }^{-/-}$, End ${ }^{+/+}$; day three, $F_{(3,30)}=1.2$, NS; day four, $F_{(3,30)}=2.9, p=0.049$, Fisher's PLSD post hoc significant difference between Enk ${ }^{+/+}$, End $^{+/+}$ and $\mathrm{Enk}^{-/-}$, End ${ }^{-/-}$, and significant difference between Enk ${ }^{+/+}$,

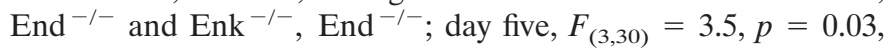

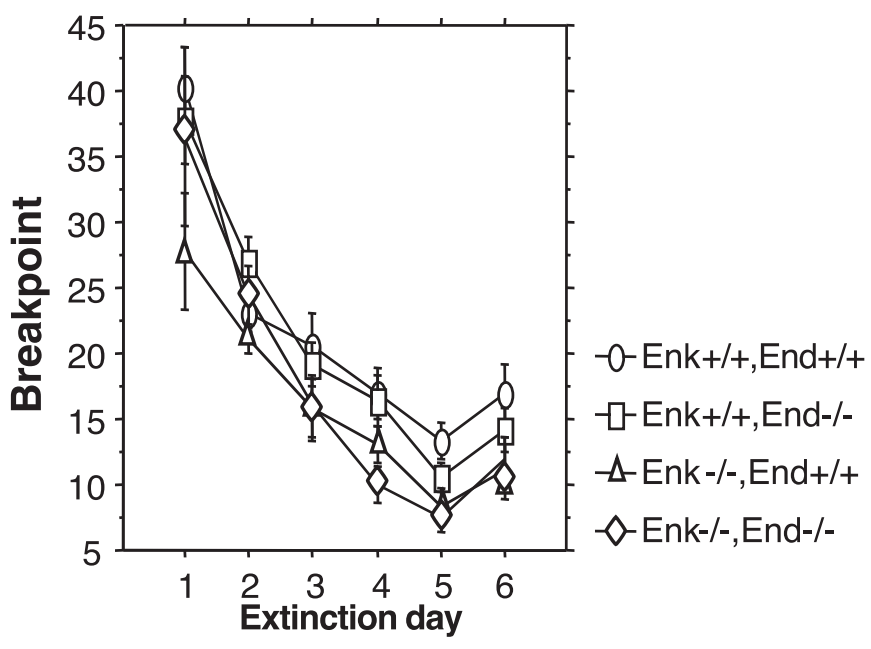

Figure 4. Extinction of operant responding under a PR3. Breakpoints $( \pm$ SEM) are given for mice under ad libitum feeding conditions for each day of extinction.
Fisher's PLSD post hoc significant difference between $\mathrm{Enk}^{+/+}$, End $^{+/+}$and Enk ${ }^{-/-}$, End ${ }^{+/+}$and significant difference between

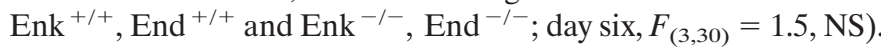
Thus, the main effect by genotype was attributable to inconsistent differences between pairs of genotypes on different days. These data confirm that resistance to extinction was equivalent among the four genotypes and was not likely a contributing factor in the reduced breakpoints of the opioid mutant mice.

Whereas breakpoints under the PR3 varied with the formula of reinforcer, we also noted an interaction between genotype and chow formula under the PR3 schedule in ad libitum fed mice. This interaction suggested that although the mutant genotypes varied their instrumental behavior for different formulas of reinforcers with the same rank order, they did not vary their response to the same degree as wild-type mice. Therefore, we determined if preference was altered in these subjects by testing consummatory behavior independently of instrumental behavior. Two-bottle free-choice experiments were conducted in the mice home cages using four concentrations of sucrose versus water and four concentrations of saccharin versus water. Sucrose and saccharin were chosen because they allow a comparison of sweet compounds with and without caloric value and because an abundant number of studies have shown that opioid antagonists will decrease preference for these two compounds. All four genotypes increased their preference for the sucrose-containing bottle with increasing concentrations in an identical pattern (Fig. $5 a$ ). We detected no main effect of genotype on the preference ratios for sucrose across four concentrations (RMANOVA, $F_{(3,31)}=0.4$, NS), but a main effect of sucrose concentration was detected (RMANOVA, $F_{(3,31)}=$ 53.2, $p<0.0001)$. Importantly, no genotype by sucrose concentration interaction was detected (RMANOVA, $F_{(9,31)}=0.6$, NS). All four genotypes modified their preference ratios similarly for the saccharin-containing bottle with increasing concentrations (Fig. $5 b$ ). We detected no main effect of genotype on the preference ratios for saccharin across four concentrations, but a main effect of saccharin concentration was detected (RMANOVA, $\left.F_{(3,31)}=180.7, p<0.0001\right)$. Importantly, no genotype by saccharin interaction was detected (RMANOVA, $F_{(9,31)}=0.9$, NS). Basal water consumption in the home cage was not different among the genotypes (data not shown).

Mice normally balance their caloric intake with energy expenditure. When a highly palatable substance like sucrose-flavored water is introduced to their diet, they will generally decrease their food intake to compensate for the extra caloric intake from sucrose. We have evidence that the male $\beta$-endorphin-deficient male mice are slightly hyperphagic between 1 and 4 months of age (S. Appleyard and M. J. Law, unpublished observations), so we determined if the caloric balance of intake was altered in the older mice used for the two-bottle free-choice experiment. Food consumption in the home cages was measured while the twobottle free-choice experiments were conducted. All four genotypes decreased their food consumption with increasing concentrations of sucrose (Fig. 6a). We detected no main effect of genotype on the amount of food eaten across four concentrations of sucrose $\left(\right.$ RMANOVA, $F_{(3,31)}=1.2$, NS), but a main effect of sucrose concentration was detected (RMANOVA, $F_{(3,31)}=33.7$, $p<0.0001)$. We also did not detect a genotype by sucrose concentration interaction (RMANOVA, $F_{(9,31)}=0.4$, NS). Using saccharin, a noncaloric tastant, we also did not detect a main effect by genotype on the amount of food eaten across four concentrations (RMANOVA, $F_{(3,31)}=1.3$, NS) (Fig. 6b). However, we detected a main effect of saccharin concentration on the amount 

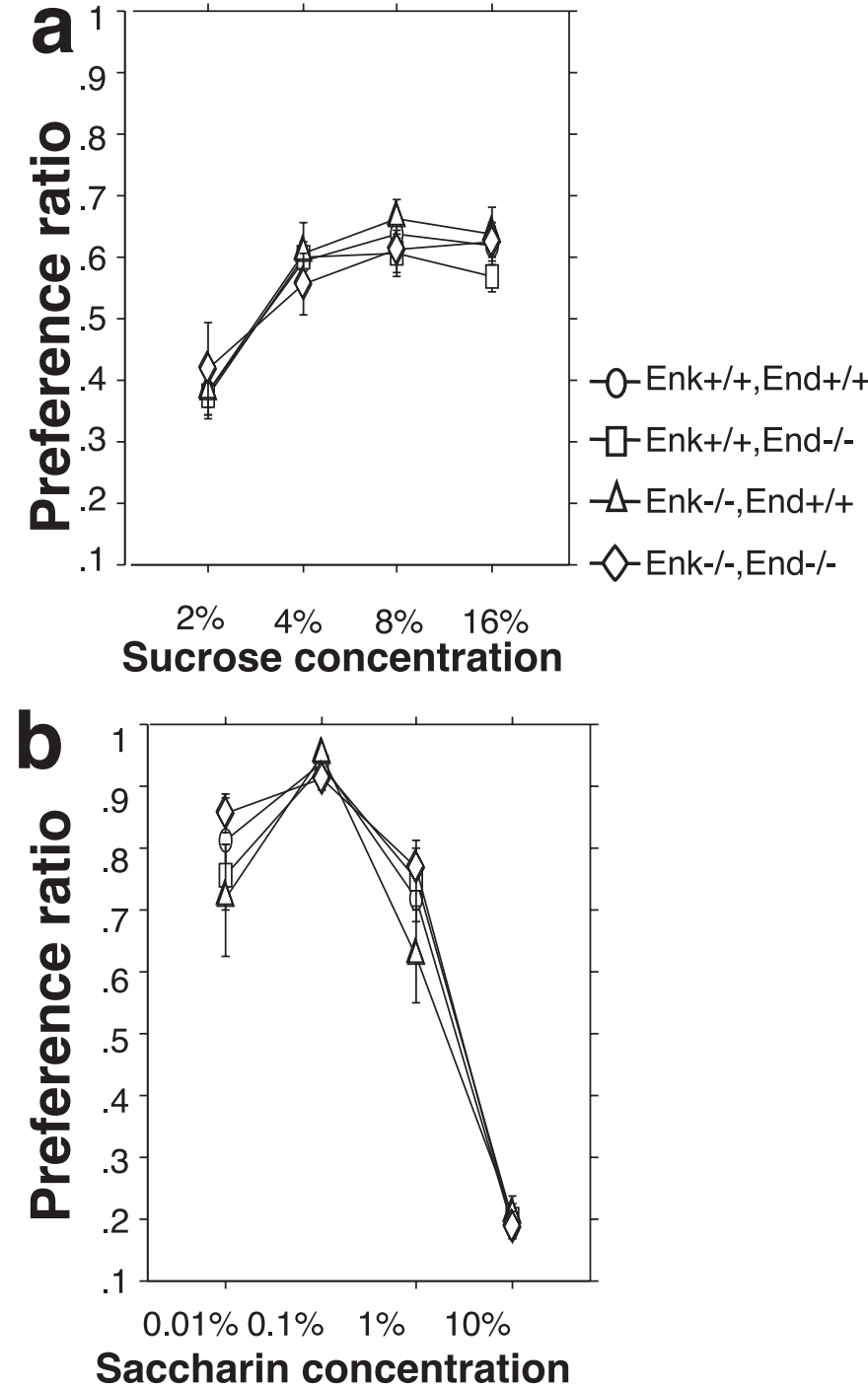

Figure 5. Two-bottle free-choice for sucrose- and saccharin-flavored water. $a$, Preference ratios $( \pm \mathrm{SEM})$ are given for four concentrations of sucrose for each of the four genotypes. $b$, Preference ratios $( \pm$ SEM) are given for four concentrations of saccharin for each of the four genotypes.

of food eaten (RMANVOA, $F_{(3,31)}=9.1, p<0.0001$ ), but no genotype by saccharin concentration interaction (RMANOVA, $F_{(9,31)}=0.4$, NS). The change in feeding during saccharin presentation was likely caused by a change in the volume of liquid consumed because the amount of food eaten did not decrease with higher concentrations of saccharin. A nonsignificant trend to increased feeding was noted in the $\mathrm{Enk}^{-/-}$, End ${ }^{-/-}$mice in both drinking experiments. Nonetheless, it appeared that regulation of energy homeostasis was primarily intact in the opioid mutant mice.

\section{DISCUSSION}

Our primary finding is that both $\beta$-endorphin- and enkephalindeficient mice have reduced breakpoints for food reinforcers when they are maintained under ad libitum feeding conditions, but not when food-restricted. These data suggest that opioids modulate the hedonic value of food independently of energy homeostasis. In addition, the opioid influence on instrumental behavior was significant regardless of the relative palatability of the reinforcers. In fact, palatability of sweet tastants in the male opioid-deficient mice

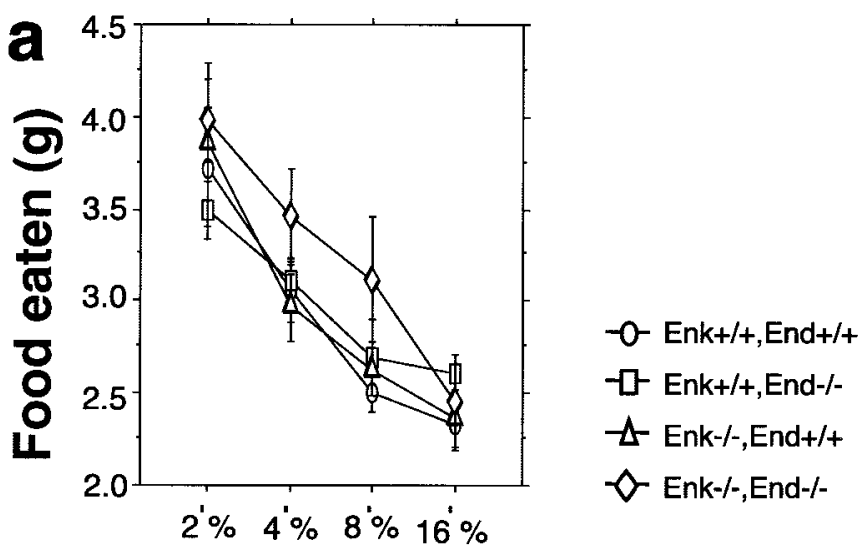

Sucrose concentration

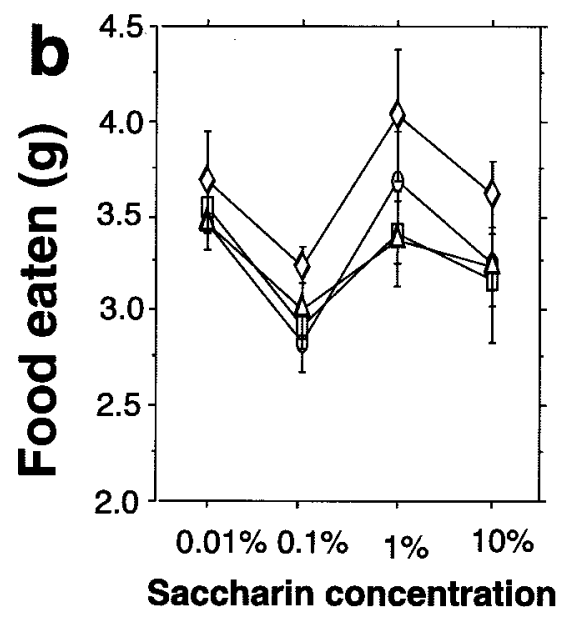

Figure 6. Food eaten per day during two-bottle free-choice tests. $a$, The amount of food eaten ( $(\mathrm{SEM})$ in the home cage during presentation of the four sucrose concentrations. $b$, The amount of food eaten $( \pm \mathrm{SEM})$ in the home cage during presentation of the four saccharin concentrations.

appeared to be unchanged when preference was measured for sucrose or saccharin. Similar findings have been reported for female enkephalin-deficient mice in response to sucrose (Ragnauth et al., 2001). We conclude that the mutant opioid genotypes have a selective reduction in the incentive value of food reinforcers revealed by instrumental behavior. These conclusions suggest that appetitive behavior (but not consummatory behavior) is modulated by $\beta$-endorphin and enkephalin, thus providing a mechanism by which endogenous opioids modulate reward related behaviors. Our findings suggest that $\beta$-endorphin and enkephalin release is not important to initiate feeding in response to caloric imbalance but rather serves to encourage feeding by nondeprived subjects by stimulating appetitive behaviors.

Both $\beta$-endorphin- and enkephalin-deficient mice had reduced breakpoints, but we did not detect an additive effect of mutations for both peptides. We were surprised that a nearly identical phenotype was observed in each of the single knock-out lines because they differ significantly in their antinociceptive behaviors. For example, stress-induced analgesia (SIA) produced by a brief room temperature forced swim was absent in $\beta$-endorphindeficient mice but intact in enkephalin-deficient mice (König et al., 1996; Rubinstein et al., 1996). Our finding here suggests that a common pathway is involved in the $\beta$-endorphin and enkephalin 
modulation of operant responding, in contrast to SIA. Previous work has indirectly implicated $\beta$-endorphin as a modulator of food-reinforced behavior (Dum et al., 1983; Dum and Herz, 1984), but the role of enkephalin in food-reinforced behavior has never been examined. $\beta$-endorphin and enkephalin may act in an interdependent manner at the receptor level. Pharmacological effects through the $\delta$ and $\mu$ opioid receptors can act synergistically, and it appears that the two receptors are physically capable of dimerizing (Gomes et al., 2000). Interactions between $\mu$ and $\delta$ receptors in vivo appear to be necessary for development of morphine tolerance because $\delta$ receptor and enkephalin knock-out mice fail to develop tolerance to the $\mu$-selective agonist (Appleyard and Low, unpublished observation) (Zhu et al., 1999). Thus, $\beta$-endorphin and enkephalin may act interdependently to modulate food-reinforced operant responding. The loss of one peptide could, then, have the same effect as the loss of both.

Although the operant behavior of opioid mutant mice is consistent with previous pharmacological studies, our results using two-bottle free choice stand in marked contrast to previous studies using opioid antagonists in rodents. Opioid receptor blockade decreased preference for sweetened liquids by rats (Lynch and Libby, 1983; Lynch, 1986; Cleary et al., 1996; Weldon et al., 1996). Genetic studies have also implicated the $\mu$ opioid receptor in positively modulating preference for saccharin in mice (Yirmiya et al., 1988). A likely possibility for these previously reported reductions in preference for sweetened liquids is an endogenous action at the $\mu$ receptor that is not $\beta$-endorphin or enkephalin mediated. Another peptide that may also act at the $\mu$ opioid receptor is dynorphin, which has long been known to stimulate feeding in rats and mice (Morley, 1987). Although this peptide is believed to act primarily at the $\kappa$ receptor, it also binds to $\mu$ receptors potently (Reisine and Pasternak, 1996), and it has been suggested that dynorphin acts through the $\mu$ receptor in at least at one brain region (Chavkin et al., 1985). Another possible endogenous opioid that could alter preference is endomorphin, which has high affinity for the $\mu$ receptor (Zadina et al., 1997) and has been shown to be orexigenic (Asakawa et al., 1998), but its ability to modulate preference has not been reported. Importantly, naloxone would block dynorphin at both the $\mu$ and $\kappa$ receptors and endomorphin at the $\mu$ receptor. Although opioid receptor subtype antagonists exist, they still cannot distinguish among endogenous peptides that bind to more than one receptor type. These points underscore the importance of examining preference and reward-related behaviors in individual peptide knock-out mice.

A developmental compensation for the lack of $\beta$-endorphin and enkephalin may have occurred, but it is unlikely that such an alteration would significantly reverse a deficit in preference for sucrose or saccharin, while leaving instrumental behavior impaired. The same compensation would have to occur independently in the $\beta$-endorphin knock-out and enkephalin knock-out strains (i.e., two distinct genes) to produce the highly similar and overlapping phenotypes we observed in the three mutant strains. However, opioid-receptor binding in the $\beta$-endorphin knock-outs does not appear to be altered for the $\mu, \kappa$, or $\delta$ receptor subtypes (Mogil et al., 2000; Slugg et al., 2000). In contrast, $\mu$ and $\delta$ receptor binding was reported to be upregulated in discrete brain regions (e.g., limbic and striatal regions) in a line of enkephalin knock-out mice different from the line used in the present study (Brady et al., 1999). Thus, changes in receptor levels are not consistent between the $\beta$-endorphin and enkephalin knock-out mice. Attributing a shared phenotype of the $\beta$-endorphin and enkephalin knock-out mice to a common compensatory change also seems improbable because the nociceptive phenotypes of the $\beta$-endorphin and enkephalin knock-out mice are quite distinct (for review, see Hayward and Low, 1999).

$\beta$-endorphin and enkephalin appear to modulate the magnitude of a conditioned response while not altering preference in a nonconditioned test. The free-choice paradigm is primarily a measurement of consummatory behavior, whereas operant responding is a measure of appetitive behavior. Thus, $\beta$-endorphin and enkephalin may modulate reward-associated behaviors independently of consumption of the positive reinforcer. In support of this interpretation is the finding that conditioned stimuli produce endorphinergic activity in the absence of consumption of a nonconditioned food stimulus (Dum and Herz, 1984). An alternative explanation for the deficit in operant responding could be that the mutants do not perseverate on the active lever, whereas wild-types do. This explanation is unlikely because all four genotypes had essentially identical extinction curves. In fact, our extinction data demonstrated that presence of the reinforcer was required to produce the observed phenotype. Additionally, under food-deprived conditions no difference in responding existed among the genotypes, so any artifact of perseveration would have to selectively confound our observation in the ad libitum feeding condition. Therefore, presence of the reinforcer may allow the subject to evaluate the reinforcer, and $\beta$-endorphin and enkephalin may be involved in the valuation of the reinforcer during conditioned behavior.

Recent work from our laboratory has demonstrated that young male $\beta$-endorphin-deficient mice are $\sim 15 \%$ heavier than their wild-type siblings (Appleyard and Low, unpublished observations). That phenotype was also evident in the subjects from this study, but the PR3 experiments were conducted on subjects over 4 months old, when the hyperphagia and difference in rate of weight gain and fat deposition were no longer present. When rodents consume a high caloric, palatable substance, such as in the sucrose study presented here, they reduce their feeding to balance their daily total caloric intake. It is worth noting that the $\beta$-endorphin-deficient mice in this study were able to normally regulate their total caloric intake during the sucrose preference test by decreasing the amount of food they ate with increasing sucrose consumption. Thus, it appears that the older $\beta$-endorphin-deficient mice are able to modify their caloric intake appropriately, which is not the case for the leptin-deficient $o b / o b$ strain (Coleman and Hummel, 1973) or melanocortin-4 receptor-deficient mice (Huszar et al., 1997). This finding is especially interesting because $\beta$-endorphin and $\alpha$-melanocyte stimulating hormone $(\alpha-\mathrm{MSH})$ are both products of the same prohormone (POMC) and are likely coreleased. A possible cooperative function of these two hormones is that $\alpha$-MSH mediates the termination of feeding, whereas $\beta$-endorphin signals the positive hedonic value of food. A modest trend to increased food consumption was noted in the $\left(\mathrm{Enk}^{-/-} \mathrm{End}^{-/-}\right)$ mice during this test, and we think this may reflect the modest hyperphagia measured in younger $\beta$-endorphin-deficient mice (Appleyard and Low, unpublished observations). Why an opioid peptide mutation would produce obese, hyperphagic animals that have decreased motivation to feed is puzzling indeed. We suggest that the two paradoxical phenotypes may be explained by $\beta$-endorphin actions in separate neural pathways. This seems especially likely because we have not found any weight differences in the male enkephalin-deficient compared with wild-type mice at any age (data not shown).

Although $\beta$-endorphin and enkephalin are not required for reinforced behavior, they clearly play a modulatory role. Our study demonstrates that both $\beta$-endorphin and enkephalin mod- 
ulate reinforced behavior under nondeprived conditions. Reinforcement under these conditions represents hedonia rather than a strict physiological response governing energy homeostasis $(\mathrm{Ca}-$ banac, 1979). The effort to receive a reinforcer combined with the reinforcer itself is critical in the evaluative process, and the endogenous opioid system may play a fundamental role in that evaluative process. This hypothesis will have to be addressed in future experiments by examining incentive valuation directly (Dickinson and Balleine, 1994). Incentive motivation should be increased in the food-deprived condition, but we found that the loss of endogenous opioids did not alter breakpoints in this higher motivational state. This finding is consistent with data from pharmacological experiments that have demonstrated that opioids are more effective at modulating feeding under ad libitum fed conditions (Hartig and Opitz, 1983; Lynch and Libby, 1983; Rudski et al., 1994; Weldon et al., 1996). For the endogenous opioid system to be unnecessary in an increased motivational state, distinguishable motivational structures must exist. It may be that the endogenous opioid system modulates motivation in both the deprived and nondeprived condition, but other factors that stimulate feeding in deprived subjects increase performance so much that opioid influence is masked. Alternatively, opioids may only alter the motivation to feed under nondeprived conditions, whereas other signals predominate during deprived conditions (e.g., leptin and insulin). Thus, in the nondeprived state opioids could increase valuation of food reinforcers, but in the deprived state involvement of the opioid system would be inhibited or superseded by pathways that mediate feeding in response to a caloric imbalance. This would imply the existence of discrete pathways that modulate incentive motivation under different motivational states (Nader et al., 1997). Increased motivation to feed during deprived conditions may therefore be directed predominately by nonopioid-modulated pathways in the brain. Opioid release may serve as an adaptive response to stimulate feeding in excess of immediate energy requirements. The resulting surfeit of calories would safeguard an organism against delay in the next possible feeding.

\section{REFERENCES}

Asakawa A, Inui A, Momose K, Ueno N, Fujino MA, Kasuga M (1998) Endomorphins have orexigenic and anxiolytic activities in mice. NeuroReport 9:2265-2267.

Bals-Kubik R, Herz A, Shippenberg TS (1989) Evidence that the aversive effects of opioid antagonists and $\kappa$-agonists are centrally mediated. Psychopharmacology 98:203-206.

Beluzzi JD, Stein L (1977) Enkephalin may mediate euphoria and drivereduction reward. Nature 266:556-558.

Brady LS, Herkenham M, Rothman RB, Partilla JS, König M, Zimmer AM, Zimmer A (1999) Region-specific up-regulation of opioid receptor binding in enkephalin knockout mice. Mol Brain Res 68:193-197.

Cabanac M (1979) Sensory pleasure. Q Rev Biol 54:1-29.

Chavkin C, Henriksen S, Siggins G, Bloom F (1985) Selective inactivation of opioid receptors in rat hippocampus demonstrates that dynorphin-A and -B may act on $\mu$-receptors in the CA1 region. Brain Res 331:366-370.

Cleary J, Weldon DT, O'Hare E, Billington C, Levine AS (1996) Naloxone effects on sucrose-motivated behavior. Psychopharmacology 126:110-114

Coleman DL, Hummel KP (1973) The influence of genetic background on the expression of the obese $(o b)$ gene in the mouse. Diabetologia 9:287-293.

Dickinson A, Balleine B (1994) Motivational control of goal-directed action. Anim Learn Behav 22:1-18.

Dum J, Herz A (1984) Endorphinergic modulation of neural reward systems indicated by behavioral changes. Pharmacol Biochem Behav 21:259-266.

Dum J, Gramsch C, Herz A (1983) Activation of hypothalamic $\beta$-endorphin pools by reward induced by highly palatable food. Pharmacol Biochem Behav 18:443-447.

Glass MC, Billington CJ, Levine AS (1999a) Opioids and food intake: distributed functional neural pathways? Neuropeptides 33:360-368.

Glass MC, O'Hare EO, Cleary JP, Billington CJ, Levine AS (1999b) The effect of naloxone on food-motivated behavior in the obese Zucker rat. Psychopharmacology 141:378-384.

Gomes I, Jordan BA, Gupta A, Trapaidze N, Nagy V, Devi LA (2000) Heterodimerization of $\mu$ and $\delta$ opioid receptors: a role in opiate synergy. J Neurosci 20:RC110.

Hartig U, Opitz K (1983) The influence of the $\kappa$-agonist bremazocine on ingestive behavior in mice and rats. Arch Int Pharmacodyn Ther 262:4-12.

Hayward MD, Low MJ (1999) Targeted mutagenesis of the murine opioid system. In: Regulatory peptides and cognate receptors (Richter D, ed), pp 169-186. Berlin: Springer.

Hayward MD, Low MJ (2001) The effect of naloxone on operant behavior for food reinforcers in DBA/2 mice. Brain Res Bull 56:537-543.

Hodos W (1961) Progressive ratio as a measure of reward strength. Science 134:943-944.

Huszar D, Lynch CA, Fairchild-Huntress V, Dunmore JH, Fang Q, Berkemeier LR, Gu W, Kesterson RA, Boston BA, Cone RD, Smith FJ, Campfield LA, Burn P, Lee F (1997) Targeted disruption of the melanocortin-4 receptor results in obesity in mice. Cell 88:131-141.

König M, Zimmer AM, Steiner H, Holmes PV, Crawley JN, Brownstein MJ, Zimmer A (1996) Pain responses, anxiety, and aggression in mice deficient in pre-proenkephalin. Nature 383:535-538.

Lynch WC (1986) Opiate blockade inhibits saccharin intake and blocks normal preference acquisition. Pharmacol Biochem Behav 24:833-836.

Lynch WC, Libby L (1983) Naloxone suppresses intake of highly preferred saccharin solutions in food deprived and sated rats. Life Sci 99:1909-1914.

Mogil JS, Grisel JE, Hayward MD, Rubinstein M, Belknap JK, Low MJ (2000) Disparate spinal and supraspinal opioid antinociceptive responses in $\beta$-endorphin-deficient mutant mice. Neuroscience 101: $709-717$.

Morley JE (1987) Neuropeptide regulation of appetite and weight. Endocrine Rev 8:256-287.

Mucha RF, Herz A (1985) Motivational properties of $\kappa$ and $\mu$ opioid receptor agonists studied with place and taste preference conditioning. Psychopharmacology 86:274-280.

Nader K, Bechara A, van der Kooy D (1997) Neurobiological constraints on behavioral models of motivation. Annu Rev Psychol 48:85-114

Ragnauth A, Schuller A, Morgan M, Chan J, Ogawa S, Pintar J, Bodnar RJ, Pfaff DW (2001) Female preproenkephalin-knockout mice display altered emotional responses. Proc Natl Acad Sci USA 98:1958-1963.

Reisine T, Pasternak G (1996) Opioid analgesics and antagonists. In: Goodman and Gilman's The pharmacological basis of therapeutics, Ed 9 (Hardman JG, Gilman AG, Limbird LE, eds), pp 521-555. New York: McGraw-Hill.

Rubinstein M, Mogil JS, Jápon M, Chan EC, Allen RG, Low MJ (1996) Absence of opioid stress-induced analgesia in mice lacking $\beta$-endorphin by site-directed mutagenesis. Proc Natl Acad Sci USA 93:2577-2582.

Rudski JM, Billington CJ, Levine AS (1994) Naloxone's effects on operant responding depend upon level of deprivation. Pharmacol Biochem Behav 49:377-383.

Slugg RM, Hayward MD, Ronnekliev OK, Low MJ, Kelly MJ (2000) Effect of the $\mu$-opioid agonist DAMGO on medial basal hypothalamic neurons in $\beta$-endorphin knock-out mice. Neuroendocrinology 72:208-217.

Székely JI (1994) $\mu$-Agonist induced euphoria as opposed to dysphoria elicited by $\kappa$-agonists in humans and experimental animals. In: Opioid peptides in substance abuse, pp 55-80. Boca Raton, FL: CRC.

Trujillo KA, Beluzzi JD, Stein L (1989) Opiate antagonists and selfstimulation: extinction-like response patterns suggest selective reward deficit. Brain Res 492:15-28.

Van Ree JM, Gerrits MAFM, Vandershuren LJMJ (1999) Opioids, reward, and addiction: An encounter of biology, psychology, and medicine. Pharmacol Rev 51:341-395.

Weldon DT, O'Hare E, Cleary J, Billington CJ, Levine AS (1996) Effect of naloxone on intake of cornstarch, sucrose, and polycose diets in restricted and nonrestricted rats. Am J Physiol 270:R1183-R1188.

Yirmiya R, Lieblich I, Liebeskind JC (1988) Reduced saccharin preference in CXBK (opioid receptor-deficient) mice. Brain Res 438: 339-342.

Zadina JE, Hackler L, Ge LJ, Kastin AJ (1997) A potent and selective endogenous agonist for the $\mu$-opiate receptor. Nature 386:499-502.

Zhu Y, King MA, Schuuler AGP, Nitsche JF, Reidl M, Elde RP, Unterwald E, Pasternak GW, Pintar JE (1999) Retention of supraspinal $\delta$-like analgesia and loss of morphine tolerance in $\partial$ opioid receptor knockout mice. Neuron 24:243-252. 